\title{
Über das Diagonalenverhältnis bei Sehnenvierecken oder: Ein Analogon zum Satz des Ptolemaios
}

Harald Riede

\begin{abstract}
Harald Riede studierte Mathematik und Physik an der Universität Mainz und promovierte 1977 an der Universität Wuppertal im Bereich der Funktionalanalysis. Nach Jahren im Schuldienst habilitierte er sich, wechselte an die Universität Eichstätt und wurde 1993 an die Universität Koblenz-Landau berufen. Seine Interessen sind weit gespannt; besondere Neigungen bestehen zu Analysis/Funktionalanalysis und Zahlentheorie.
\end{abstract}

Genau die Sehnenvierecke (= Kreisvierecke) haben unter den konvexen Vierecken die Eigenschaft

$$
e \cdot f=a c+b d
$$

dabei sind $a, b, c, d$ die Seiten, $e, f$ die Diagonalen gemäß Fig. 1. Dies ist der bekannte Satz des Ptolemaios, der das Produkt der Diagonalen durch die Seitenlängen ausdrückt.

Es gilt nun eine ganz entsprechende Aussage über den Quotienten der Diagonalen:

Satz Für ein konvexes Viereck gilt

$$
\frac{e}{f}=\frac{a d+b c}{a b+c d}
$$

genau dann, wenn es sich um ein Sehnenviereck handelt.

Vielen Lesern dürfte der Satz des Ptolemaios aus der Elementargeometrie bekannt sein: Ein Sehnenviereck mit den Seiten $a, b, c, d$ und den Diagonalen $e, f$ wird durch die Beziehung $e f=a c+b d$, d.h. das Diagonalenprodukt ist gleich der Summe der Gegenseitenprodukte, charakterisiert. Im Gegensatz dazu wird in der vorliegenden Arbeit eine Charakterisierung gegeben, die den Quotienten $e / f$ heranzieht. Auf elementare Weise wird gezeigt, dass ein konvexes Viereck genau dann ein Sehnenviereck ist, wenn die Relation $e / f=(a d+b c) /(a b+c d)$ gilt. Obgleich diese Beziehung für Sehnenvierecke schon lange bekannt ist, scheint die Charakterisierung von Sehnenvierecken durch sie neu zu sein. 


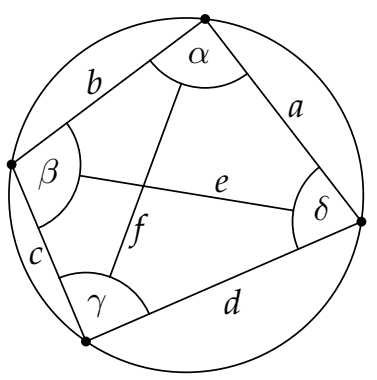

Fig. 1

Ich danke hier zunächst meinem Kollegen S. Deschauer (Dresden), der mich durch sachkundige Literaturhinweise vor der (zugegebenermaßen naiven) Annahme bewahrt hat, etwa ich sei der Entdecker dieses Satzes. Tatsache ist (vgl. Tropfke [6, 153-164]): Die Eigenschaft (2) wurde für Sehnenvierecke schon von Brahmagupta (598-668) behauptet, erstmals 1464 von Regiomontan, dann bald auch (eleganter) von anderen abgeleitet (etwa J. Praetorius, W. Snellius, 1598). Für die Umkehrung allerdings, wie sie im Satz mitformuliert ist, scheint der Nachweis bisher noch auszustehen; nur soviel ist mir jetzt bekannt: M.G. Fontené nimmt 1908 in [3] ihre Gültigkeit an, bleibt den Beweis aber schuldig. Diesem Mangel wird im Folgenden abgeholfen.

Der obige Satz ist in seinen Spezialfällen natürlich nur da von Wert, wo die Diagonalen $e$, $f$ nicht schon aus Symmetriegründen gleich lang sind, wie dies beim gleichschenkligen Trapez der Fall ist (hier liefert (1) immerhin den Babylonischen Diagonalensatz; zu diesem vgl. etwa: Hofmann [4]).

Mittels (1) und (2) lassen sich $e, f$ natürlich unmittelbar allein durch die Seiten ausdrücken. So ist beispielsweise $e^{2}=(a c+b d)(a d+b c) /(a b+c d)$.

Die Voraussetzung der Konvexität ist für den Satz unverzichtbar, denn man findet ohne Mühe nicht-konvexe Vierecke mit der Eigenschaft (2), die keine Sehnenvierecke sind. Für sie gilt nach Desboves [1] und Fontené [3]:

$$
\left(a^{2}+b^{2}+c^{2}+d^{2}-e^{2}-f^{2}\right)(a c+b d+e f)=2(a b+c d)(a d+b c),
$$

speziell etwa für gegebenes $a=b>c=d=1$ mit einspringender Ecke $D$ (Deltadrachen):

$$
e=\frac{2 a\left(a^{2}-1\right)}{\sqrt{a^{6}+7 a^{4}+7 a^{2}+1}}, \quad f=\frac{a^{4}-1}{\sqrt{a^{6}+7 a^{4}+7 a^{2}+1}} .
$$

Bevor ich den allgemeinen Beweis antrete, sei als „,vertrauensbildende Maßnahme“ ein Fall behandelt, in dem (2) vereinfacht auftritt.

Für einen Kreisdrachen, etwa mit $a=b, c=d$, wird (1) zu ef $=2 a c$, (2) zu $\frac{e}{f}=\frac{2 a c}{a^{2}+c^{2}}$, was wegen $f^{2}=a^{2}+c^{2}$ auf dasselbe hinausläuft.

Ist umgekehrt ein Drachen $a=b, c=d$ mit $\alpha=90^{\circ}$ gegeben, ohne Beschränkung der Allgemeinheit mit $a=1$ (also $e=\sqrt{2}$ ), so besagt (2): $f=e \cdot \frac{1+c^{2}}{2 c}$. In dem durch $e$, 
$c, f$ begrenzten rechtwinkligen Dreieck gilt dann

$$
\left(\frac{\sqrt{2}}{2}\right)^{2}+\left(f-\frac{\sqrt{2}}{2}\right)^{2}=c^{2}, \quad c^{2}+\left(1+c^{2}-c\right)^{2}-2 c^{4}=0
$$

folglich $(c-1)\left(c^{3}+3 c^{2}-c+1\right)=0$. Die einzige positive Lösung ist $c=1$, die Figur also ein Kreisdrachen.

\section{Der allgemeine Beweis}

Gegeben sei ein konvexes Sehnenviereck, d.h. $\gamma=180^{\circ}-\alpha$ (Fig. 1). Nach dem Kosinussatz ist

$$
e^{2}=a^{2}+b^{2}-2 a b \cos \alpha=c^{2}+d^{2}+2 c d \cos \alpha,
$$

folglich

$$
c d e^{2}+a b e^{2}=c d\left(a^{2}+b^{2}\right)+a b\left(c^{2}+d^{2}\right)
$$

nach (1) also

$$
e^{2}(a b+c d)=(a c+b d)(a d+b c)=e f(a d+b c) .
$$

Will man den „Ptolemaios“ bei der Herleitung vermeiden, bietet sich folgendes Vorgehen an (Fig. 2): Bezeichnet $F$ die Fläche des Sehnenvierecks, so folgt aus

$$
2 F=a b \sin \gamma+c d \sin \gamma=(a b+c d) \sin \gamma, \quad 2 F=a d \sin \delta+b c \sin \delta=(a d+b c) \sin \delta
$$

sofort mit dem Sinussatz

$$
\frac{e}{f}=\frac{e}{d} \cdot \frac{d}{f}=\frac{\sin \gamma}{\sin \varphi} \cdot \frac{\sin \varphi}{\sin \delta}=\frac{a d+b c}{a b+c d} .
$$

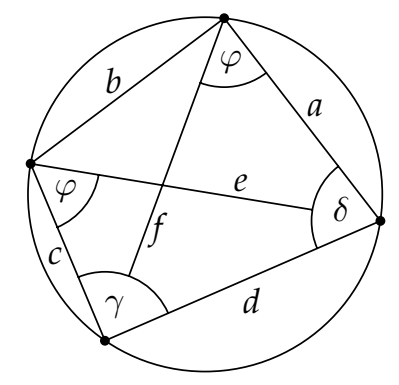

Fig. 2

Nun zur umgekehrten Richtung. Sei ein konvexes Viereck $a, \ldots, f$ gegeben, das der Bedingung (2) genügt. Wir entfernen die starren Diagonalen und stellen uns das Ganze als Gelenkviereck vor, also mit beweglichen Winkeln (s. Fig. 3). Nach Voraussetzung besitzt dieses Gestänge mindestens eine Stellung, in der (2) erfüllt ist, in der also das Verhältnis $\frac{e}{f}$ gerade den (während der Bewegung des Gelenkvierecks festen) Wert $(a d+b c) /(a b+c d)$ annimmt. 
Ohne Beschränkung der Allgemeinheit kann $a+b \leq c+d, b+c \leq a+d$ angenommen werden (andernfalls Umbenennung: etwa im Fall $a+b>c+d$ werden $a, d$ und $b, c$ wechselseitig umbenannt; das hat keinen Einfluss auf (2)! Anschaulich entspricht dies einem Umklappen des Vierecks längs $e$ ). Wir zeigen für abnehmendes $e$ (und notwendig wachsendes $f$ ) einige mögliche Konstellationen für das Viereck:

a)

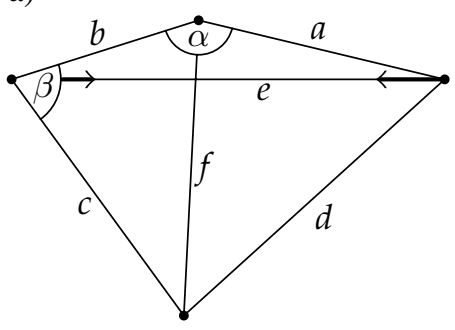

c)

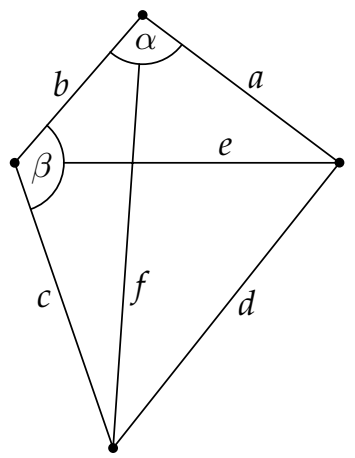

b)

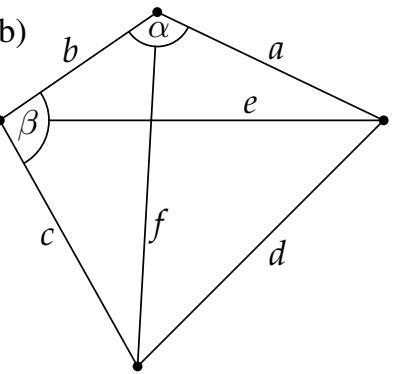

d)

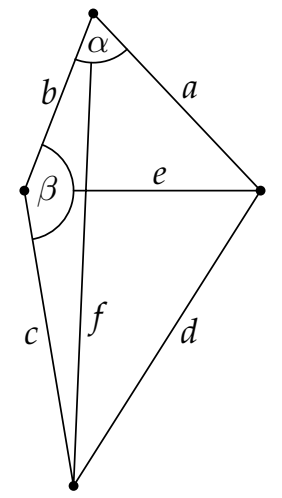

Fig. 3

Man erkennt, dass sich von a) nach d) gewisse Veränderungen streng monoton vollziehen: Mit $e$ muss auch $\alpha$ (ebenso $\gamma$ ) abnehmen, mit dann notwendig wachsendem $f$ wächst auch $\beta$. Dabei gibt es zwei Grenzlagen, die in a) und d) beinahe erreicht sind:

$$
\begin{array}{ll}
\alpha=180^{\circ}: & e=a+b \text { ist maximal, } \quad f=f_{\min } \text { ist minimal, } \\
\beta=180^{\circ}: & e=e_{\min } \text { ist minimal, } \quad f=b+c \text { ist maximal. }
\end{array}
$$

Also durchläuft dabei $\frac{e}{f}$ streng monoton fallend das offene Intervall $\left(\frac{e_{\min }}{b+c}, \frac{a+b}{f_{\min }}\right)$. Dass dann irgendwann die Übereinstimmung (2) eintritt, ist klar. Klar ist jetzt aber auch, dass aufgrund der stetigen und streng monotonen Veränderung die Beziehung (2) nur genau ein Mal gültig sein kann.

Die Summe $\alpha+\gamma$ ist ,anfangs“ maximal und $>180^{\circ}$ (da im Grenzfall schon $\alpha=180^{\circ}$ ); am „Ende“ der oben beschriebenen Deformation nimmt $\alpha+\gamma$ seinen kleinstmöglichen 
Wert an und ist dann $<180^{\circ}$ (da im Grenzfall schon $\beta=180^{\circ}$ wird). Also hat $\alpha+\gamma$ irgendwann zwischendrin den Wert $180^{\circ}$. Dann ist aber das Viereck ein Sehnenviereck und es gilt (nach dem ersten Beweisteil) die Gleichung (2). Da (2), wie oben begründet, nur genau ein Mal angenommen wird, liegt damit der Fall des ursprünglich gegebenen Vierecks vor.

Damit ist der Beweis komplett. Wer für den zweiten Teil eine rein rechnerische Argumentation zuwege bringen will, muss sich bei seinem Bemühen, gelinde gesagt, auf einige Schwierigkeiten einstellen. Darin liegt wohl auch der Grund, weshalb Fontené es in [3] bei der reinen Behauptung belässt.

Eine weiterführende Aufgabe

Aus der für beliebige konvexe Vierecke gültigen Beziehung (siehe etwa Schuler [5])

$$
e^{2} f^{2}=a^{2} c^{2}+b^{2} d^{2}-2 a b c d \cos (\alpha+\gamma)
$$

folgt (1), der Ptolemaische Lehrsatz, unmittelbar. Man finde eine entsprechende Verallgemeinerung $\frac{e^{2}}{f^{2}}=\ldots$ für (2)! Sie könnte im Übrigen hilfreich sein bei dem gerade genannten Problem des rechnerischen Rückbeweises.

\section{Literatur}

[1] Desboves, M.: Lettre à M. Brisse. Nouvelles annales de mathématique 16 (1877) 2, 226.

[2] Encyklopädie der Mathematischen Wissenschaften, Bd. 3 (Geometrie), Leipzig 1914-1931; hierin: M. Zacharias: Elementargeometrie, 996.

[3] Fontené, M.G.: Sur les Quadrangles de Desboves. Nouvelles annales de mathématique 8 (1809) 4, 16.

[4] Hofmann, J.E.: Geistreiche geometrische Konstruktionen im Wandel der Zeiten. Der Mathematikunterricht 3 (1964), 7-47.

[5] Schuler, K.: Verallgemeinerung des Satzes von Ptolemaios. Der Mathematikunterricht 1 (1968), 63-68.

[6] Tropfke, J.: Geschichte der Elementarmathematik. Bd. IV (Ebene Geometrie), Berlin 1939.

Harald Riede

Mathematisches Institut

Universität Koblenz-Landau

Universitätsstraße 1

D-56070 Koblenz, Deutschland

e-mail: riede@uni-koblenz.de 